\title{
UNIT-CIRCLE-PRESERVING MAPPINGS
}

\section{SOON-MO JUNG and BYUNGBAE KIM}

\author{
Received 6 April 2004
}

We prove that if a one-to-one mapping $f: \mathbb{R}^{n} \rightarrow \mathbb{R}^{n}(n \geq 2)$ preserves the unit circles, then $f$ is a linear isometry up to translation.

2000 Mathematics Subject Classification: 51K05.

1. Introduction. Let $X$ and $Y$ be normed spaces. A mapping $f: X \rightarrow Y$ is called an isometry if $f$ satisfies the equality

$$
\|f(x)-f(y)\|=\|x-y\|
$$

for all $x, y \in X$. A distance $r>0$ is said to be preserved (conserved) by a mapping $f: X \rightarrow Y$ if

$$
\|f(x)-f(y)\|=r \quad \forall x, y \in X \text { with }\|x-y\|=r .
$$

If $f$ is an isometry, then every distance $r>0$ is conserved by $f$, and vice versa. We can now raise a question whether each mapping that preserves certain distances is an isometry. Indeed, Aleksandrov [1] had raised a question whether a mapping $f: X \rightarrow X$ preserving a distance $r>0$ is an isometry, which is now known to us as the Aleksandrov problem. Without loss of generality, we may assume $r=1$ when $X$ is a normed space (see [16]).

Beckman and Quarles [2] solved the Aleksandrov problem for finite-dimensional real Euclidean spaces $X=\mathbb{R}^{n}$ (see also $[3,4,5,6,7,8,11,12,13,14,15,17,18,19,20]$ ).

THEOREM 1.1 (Beckman and Quarles). If a mapping $f: \mathbb{R}^{n} \rightarrow \mathbb{R}^{n}(2 \leq n<\infty)$ preserves a distance $r>0$, then $f$ is a linear isometry up to translation.

Recently, Zaks [25] proved the rational analogues of the Beckman-Quarles theorem. Indeed, he assumes that $n=4 k(k+1)$ for some $k \geq 1$ or $n=2 m^{2}-1$ for some $m \geq 3$, and he proves that if a mapping $f: \mathbb{Q}^{n} \rightarrow \mathbb{Q}^{n}$ preserves the unit distance, then $f$ is an isometry (see also [21, 22, 23, 24]).

It seems interesting to investigate whether the "distance $r>0$ " in the BeckmanQuarles theorem can be replaced by some properties characterized by "geometrical figures" without loss of its validity.

In [9], the first author proved that if a one-to-one mapping $f: \mathbb{R}^{n} \rightarrow \mathbb{R}^{n}(n \geq 2)$ maps every regular triangle (quadrilateral or hexagon) of side length $a>0$ onto a figure of 


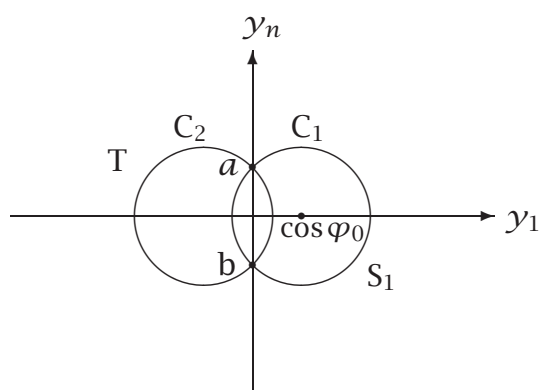

FIGURE 2.1

the same type with side length $b>0$, then there exists a linear isometry $I: \mathbb{R}^{n} \rightarrow \mathbb{R}^{n}$ up to translation such that

$$
f(x)=\frac{b}{a} I(x) .
$$

Furthermore, the first author proved that if a one-to-one mapping $f: \mathbb{R}^{2} \rightarrow \mathbb{R}^{2}$ maps every unit circle onto a unit circle, then $f$ is a linear isometry up to translation (see [10]).

In this connection, we will extend the result of [10] to the $n$-dimensional cases; more precisely, we prove in this paper that if a one-to-one mapping $f: \mathbb{R}^{n} \rightarrow \mathbb{R}^{n}(n \geq 2)$ maps every unit circle onto a unit circle, then $f$ is a linear isometry up to translation.

2. Preliminaries. We start with any two distinct points $a$ and $\mathrm{b}$ in $\mathbb{R}^{n}$ with the distance between the two less than 2 . Let their distance be

$$
2 c=2 \sin \varphi_{0} \quad \text { with } 0<\varphi_{0}<\frac{\pi}{2}, 0<c<1 .
$$

Given such two distinct points whose distance is less than 2, we can choose a coordinate $\left(y_{1}, \ldots, y_{n}\right)$ for $\mathbb{R}^{n}$ such that

$$
a=\left(0, \ldots, 0, \sin \varphi_{0}\right), \quad \mathrm{b}=\left(0, \ldots, 0,-\sin \varphi_{0}\right) .
$$

Let the $(n-2)$-dimensional unit sphere contained in the space orthogonal to the $y_{n^{-}}$ direction be

$$
\mathrm{Y}=\left\{\left(y_{1}, \ldots, y_{n-1}, 0\right) \mid y_{1}^{2}+\cdots+y_{n-1}^{2}=1\right\}
$$

If we call the center of any unit circle passing through the two points ( $a$ and $b$ ) o' and the origin of the coordinate o, then the vector $\overrightarrow{\mathrm{oo}}^{\prime}$ is perpendicular to the $y_{n}$-axis and its length must be $\cos \varphi_{0}$ and therefore $\overrightarrow{\mathrm{oo}^{\prime}} \in \tilde{\mathrm{Y}}=\cos \varphi_{0} \mathrm{Y}$, see Figure 2.1. It means that any unit circle passing through the points $a$ and $\mathrm{b}$ has its center in $\tilde{Y}=\cos \varphi_{0} \mathrm{Y}$. Let $\mathrm{T}$ be the set of union of all the unit circles passing through the points $a$ and $\mathrm{b}$. More precisely, if we define the following set:

$$
\mathrm{T}=\left\{\left(\cos \varphi+\cos \varphi_{0}\right) y+(0, \ldots, 0, \sin \varphi) \mid y \in \mathrm{Y}, 0 \leq \varphi<2 \pi\right\},
$$


then it is clear that this is the set of union of all the unit circles which are centered at $\cos \varphi_{0} y$ for each fixed $y \in \mathrm{Y}$ and which pass through $a$ and $\mathrm{b}$ when $\varphi=\pi \mp \varphi_{0}$ (see Figure 2.1).

The intersection of T and the $y_{1}-y_{n}$ plane consists of two circles, say $\mathrm{C}_{1}$ (when $y_{1}=1$, i.e., $y=(1,0, \ldots, 0)$ ) and $\mathrm{C}_{2}$ (when $y_{1}=-1$, i.e., $y=(-1,0, \ldots, 0)$, see Figure 2.1$)$. In the following contexts, we will consider the cases $y_{1}=1$ and -1 in connection with $\mathrm{T}$ as the circles $C_{1}$ and $C_{2}$, respectively. Call $S_{1}$ the $(n-1)$-dimensional unit sphere containing the circle $C_{1}$. If we let the center of $C_{1}$ be $O$ and the center of $S_{1}$ be $\tilde{O}$, then it is obvious that $\mathrm{O}=\tilde{\mathrm{O}}$.

(To see this, choose any point $\mathrm{A} \in \mathrm{C}_{1}$ and its antipodal point $\mathrm{B}$ in $\mathrm{C}_{1}$. Then, by the definition of the antipodal points that they lie exactly the opposite with respect to the center of the circle $C_{1}$ whose center is at $O$, and because they are of the same length 1 , we have the following condition that

$$
\overrightarrow{\mathrm{OA}}=-\overrightarrow{\mathrm{OB}}, \quad \overrightarrow{\mathrm{AB}}=\overrightarrow{\mathrm{AO}}+\overrightarrow{\mathrm{OB}}=2 \overrightarrow{\mathrm{OB}}
$$

On the other hand, we have, since the two points A and B lie also on the unit sphere $\mathrm{S}_{1}$ with its center at $\tilde{O}$,

$$
2=|\overrightarrow{\mathrm{AB}}|=|\overrightarrow{\mathrm{AO}}+\overrightarrow{\tilde{\mathrm{OB}}}| \leq|\overrightarrow{\mathrm{AO}}|+|\overrightarrow{\tilde{O} \mathrm{~B}}|=1+1=2 .
$$

Therefore, by the Cauchy-Schwarz inequality, $\overrightarrow{\mathrm{AO}}$ is a positive multiple of $\overrightarrow{\text { OB }}$, which means $\overrightarrow{A \tilde{O}}=\overrightarrow{\tilde{O} B}$ because their lengths are both 1 . So,

$$
\overrightarrow{\mathrm{AB}}=\overrightarrow{\mathrm{AO}}+\overrightarrow{\tilde{O} \mathrm{~B}}=2 \overrightarrow{\tilde{O} \mathrm{~B}}
$$

and therefore $\tilde{O}=0$.)

Now, we first show that $S_{1}$ and $T$ intersect only at $C_{1}$. To make computation simpler we use a new coordinate $x$ for $\mathbb{R}^{n}$, where

$$
x=y-\left(\cos \varphi_{0}, 0, \ldots, 0\right) .
$$

In this coordinate (see Figure 2.2), $\mathrm{S}_{1}$ becomes the unit sphere $S$ centered at the origin,

$$
\begin{aligned}
& \mathrm{S}_{1}=S=\left\{\left(x_{1}, \ldots, x_{n}\right) \mid x_{1}^{2}+\cdots+x_{n}^{2}=1\right\}, \\
& \mathrm{T}=\left\{x=\left(\cos \varphi+\cos \varphi_{0}\right) y+(0, \ldots, 0, \sin \varphi)\right. \\
& \left.\quad-\left(\cos \varphi_{0}, 0, \ldots, 0\right) \mid y \in \mathrm{Y}, 0 \leq \varphi<2 \pi\right\} .
\end{aligned}
$$

With the help of this coordinate we show the following lemma.

LEMMA 2.1. $\mathrm{T} \cap \mathrm{S}_{1}=\mathrm{C}_{1}$. 

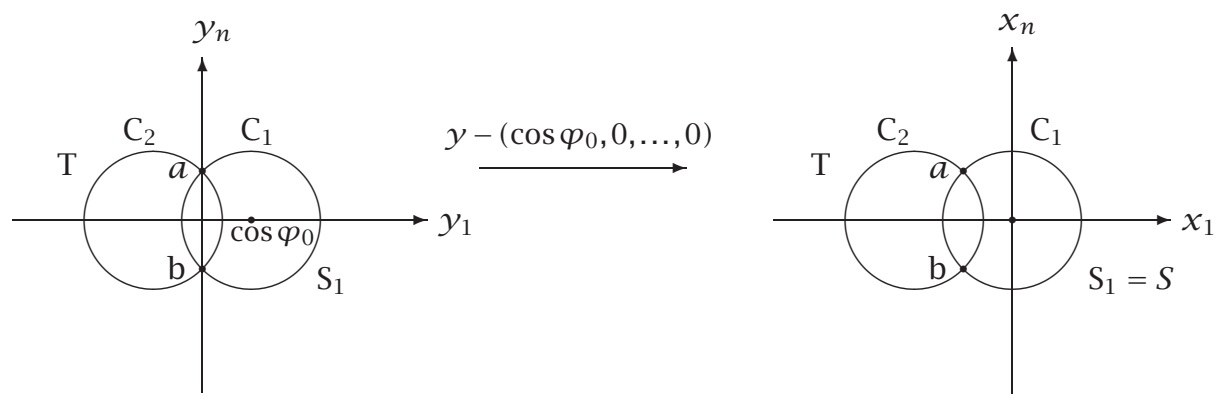

FIGURE 2.2

Proof. If any element in T has distance 1 from the origin of the $x$-coordinate, then we have

$$
\begin{aligned}
1= & {\left[\left(\cos \varphi+\cos \varphi_{0}\right) y_{1}-\cos \varphi_{0}\right]^{2}+\left(\cos \varphi+\cos \varphi_{0}\right)^{2} y_{2}^{2} } \\
& +\cdots+\left(\cos \varphi+\cos \varphi_{0}\right)^{2} y_{n-1}^{2}+\sin ^{2} \varphi \\
= & \left(\cos \varphi+\cos \varphi_{0}\right)^{2}-2 \cos \varphi_{0}\left(\cos \varphi+\cos \varphi_{0}\right) y_{1}+\cos ^{2} \varphi_{0}+\sin ^{2} \varphi \\
= & 1+2 \cos ^{2} \varphi_{0}\left(1-y_{1}\right)+2 \cos \varphi_{0} \cos \varphi\left(1-y_{1}\right) .
\end{aligned}
$$

Therefore, we have

$$
0=2 \cos \varphi_{0}\left(1-y_{1}\right)\left(\cos \varphi+\cos \varphi_{0}\right)
$$

With $y_{1}=1$, $\mathrm{T}$ in (2.10) represents the unit circle $\mathrm{C}_{1}$ in the $x_{1}-x_{n}$ plane. If

$$
\cos \varphi=-\cos \varphi_{0}, \quad \text { i.e., } \varphi=\pi \mp \varphi_{0},
$$

then it follows from (2.10) that

$$
\mathrm{T}=\left\{x=\left(-\cos \varphi_{0}, 0, \ldots, 0, \pm \sin \varphi_{0}\right)\right\}=\{a, \mathrm{~b}\}
$$

which also belong to $\mathrm{C}_{1}$.

Now, consider, as in Figure 2.3, the origin e and $\tilde{\mathrm{e}}=(-2,0, \ldots, 0)$ in the $x$-coordinate and the unit circle $\mathrm{C}_{1}$ passing through e and ẽ in the $x_{1}-x_{n}$ plane. Choose a point $\mathrm{d} \in \mathrm{C}_{1}$, $\mathrm{d} \notin\{\mathrm{e}, \tilde{\mathrm{e}}\}$. We parameterize all the unit circles passing through the points e and $\mathrm{d}$. We assume the $x_{n}$-coordinate of $\mathrm{d}$ is negative.

By triangle inequality, the distance between e and d is less than 2 , say $2 \sin \varphi_{0}$, with $0<\varphi_{0}<\pi / 2$. Choose a new coordinate $y$ for $\mathbb{R}^{n}$ and consider two points

$$
\mathrm{e}^{\prime}=\left(0, \ldots, 0, \sin \varphi_{0}\right), \quad \mathrm{d}^{\prime}=\left(0, \ldots, 0,-\sin \varphi_{0}\right),
$$

(see Figure 2.4). 


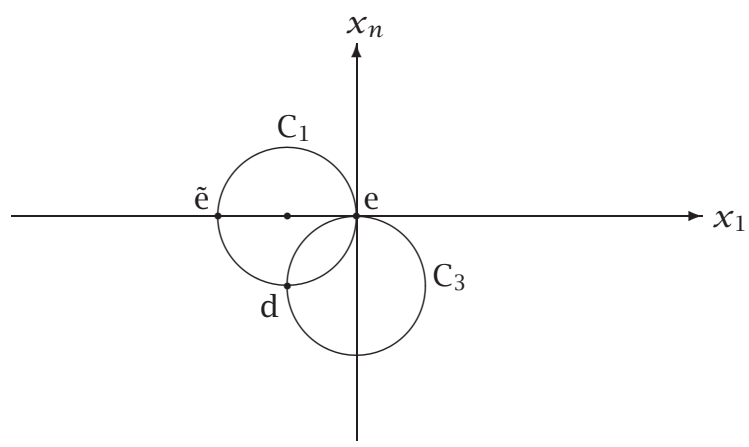

FIGURE 2.3
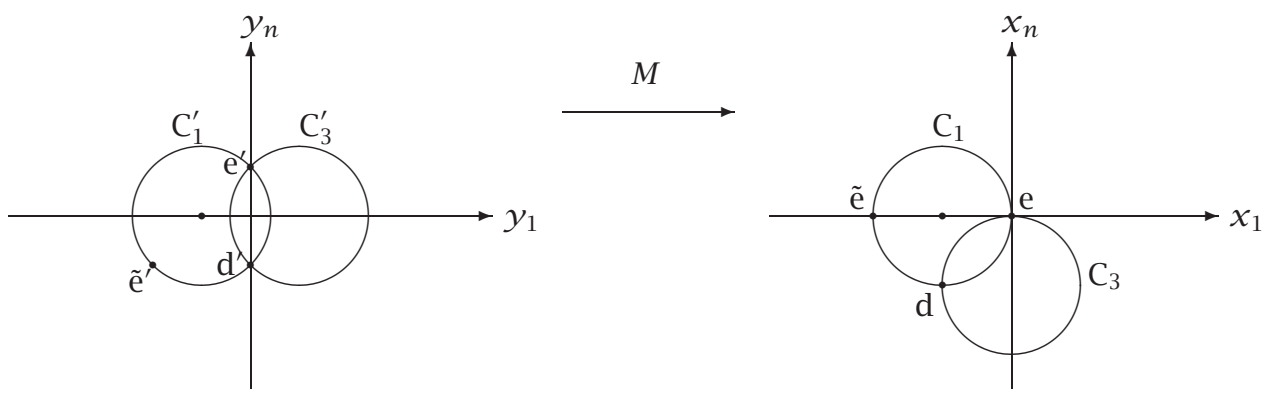

FIGURE 2.4

To get a parameterization of the unit circles passing through e and d, we consider the mapping $M$ defined by

$$
x=M y=\left[\begin{array}{ccccc}
\cos \varphi_{0} & 0 & \cdots & 0 & \sin \varphi_{0} \\
0 & 1 & \cdots & 0 & 0 \\
\vdots & \vdots & \ddots & \vdots & \vdots \\
0 & 0 & \cdots & 1 & 0 \\
-\sin \varphi_{0} & 0 & \cdots & 0 & \cos \varphi_{0}
\end{array}\right]\left[y+\left(\cos \varphi_{0}, 0, \ldots, 0\right)\right]^{\mathrm{t}}-(1,0, \ldots, 0)^{\mathrm{t}}
$$

This transformation $M$ is an isometry (since it is a composition of a rotation and translations) and sends

$$
\left\{y=\left(0, \ldots, 0, \pm \sin \varphi_{0}\right)\right\}=\left\{\mathrm{e}^{\prime}, \mathrm{d}^{\prime}\right\}
$$

to

$$
\left\{x=(0, \ldots, 0), x=\left(\cos \left(-2 \varphi_{0}\right)-1,0, \ldots, 0, \sin \left(-2 \varphi_{0}\right)\right)\right\}=\{\mathrm{e}, \mathrm{d}\}
$$

and therefore it sends any unit circle passing through $\mathrm{e}^{\prime}$ and $\mathrm{d}^{\prime}$ to a unit circle passing through e and d. 
Therefore, by comparing Figure 2.4 with Figure 2.1 and considering (2.4), all the unit circles passing through e and d can be parameterized as

$$
\left\{x=M y \mid y=\left(\cos \varphi+\cos \varphi_{0}\right) y^{\prime}+(0, \ldots, 0, \sin \varphi), y^{\prime} \in \mathrm{Y}, 0 \leq \varphi<2 \pi\right\}
$$

With the help of this parameterization, we are ready to show the following lemma.

LEMma 2.2. For $\mathrm{d} \in \mathrm{C}_{1}, \mathrm{~d} \notin\{\mathrm{e}, \tilde{\mathrm{e}}\}$, any unit circle in $\mathbb{R}^{n}$, which passes through $\mathrm{d}$ and $\mathrm{e}$, has some point whose $x_{1}$-coordinate is positive, except the circle $\mathrm{C}_{1}$.

Proof. Without loss of generality, we can assume the $x_{n}$-coordinate of $\mathrm{d}$ is negative. Note that with $\varphi=\pi \mp \varphi_{0}$ in (2.19), $y=\left(0, \ldots, 0, \pm \sin \varphi_{0}\right)$ are the points $\mathrm{e}^{\prime}$ or $\mathrm{d}^{\prime}$ in the $y$-coordinate and further $\varphi=\pi \mp \varphi_{0}$ means that

$$
x=(0, \ldots, 0)=\mathrm{e}, \quad x=\left(\cos \left(-2 \varphi_{0}\right)-1,0, \ldots, 0, \sin \left(-2 \varphi_{0}\right)\right)=\mathrm{d}
$$

in the $x$-coordinate, regardless of $y^{\prime} \in \mathrm{Y}$. Any unit circle passing through e and $\mathrm{d}$ is given as $x=M y$ with $y$ given as in (2.19), that is,

$$
\left[\begin{array}{c}
x_{1} \\
x_{2} \\
\vdots \\
x_{n-1} \\
x_{n}
\end{array}\right]=\left[\begin{array}{ccccc}
\cos \varphi_{0} & 0 & \cdots & 0 & \sin \varphi_{0} \\
0 & 1 & \cdots & 0 & 0 \\
\vdots & \vdots & \ddots & \vdots & \vdots \\
0 & 0 & \cdots & 1 & 0 \\
-\sin \varphi_{0} & 0 & \cdots & 0 & \cos \varphi_{0}
\end{array}\right]\left[\begin{array}{c}
\left(\cos \varphi+\cos \varphi_{0}\right) y_{1}^{\prime}+\cos \varphi_{0} \\
\left(\cos \varphi+\cos \varphi_{0}\right) y_{2}^{\prime} \\
\vdots \\
\left(\cos \varphi+\cos \varphi_{0}\right) y_{n-1}^{\prime} \\
\sin \varphi
\end{array}\right]-\left[\begin{array}{c}
1 \\
0 \\
\vdots \\
0 \\
0
\end{array}\right]
$$

The first coordinate is

$$
x_{1}=\cos \varphi_{0}\left(\cos \varphi+\cos \varphi_{0}\right) y_{1}^{\prime}+\cos ^{2} \varphi_{0}+\sin \varphi_{0} \sin \varphi-1
$$

We show that for $y_{1}^{\prime} \neq-1\left(y_{1}^{\prime}=-1\right.$ means the circle $C_{1}^{\prime}$ in the $y$-coordinate and the circle $C_{1}$ in the $x$-coordinate, see Figure 2.4), there is always some $\varphi$ near $\pi-\varphi_{0}$ (i.e., near the point e) such that the above $x_{1}$ becomes positive.

Let

$$
\theta=\left(\pi-\varphi_{0}\right)-\varphi=\pi-\left(\varphi+\varphi_{0}\right)
$$

and so

$$
\varphi=\pi-\left(\theta+\varphi_{0}\right)
$$


Then, the above is

$$
\begin{aligned}
x_{1}= & -\cos \varphi_{0} \cos \left(\theta+\varphi_{0}\right) y_{1}^{\prime}+\cos ^{2} \varphi_{0}\left(1+y_{1}^{\prime}\right)+\sin \varphi_{0} \sin \left(\theta+\varphi_{0}\right)-1 \\
= & -\cos \varphi_{0}\left[\cos \theta \cos \varphi_{0}-\sin \theta \sin \varphi_{0}\right] y_{1}^{\prime}+\sin \varphi_{0}\left[\sin \theta \cos \varphi_{0}+\cos \theta \sin \varphi_{0}\right] \\
& \quad-1+\cos ^{2} \varphi_{0}\left(1+y_{1}^{\prime}\right) \\
= & \sin \theta \sin \varphi_{0} \cos \varphi_{0}\left(1+y_{1}^{\prime}\right)+\cos \theta \sin ^{2} \varphi_{0}-\cos \theta \cos ^{2} \varphi_{0} y_{1}^{\prime} \\
& \quad-1+\cos ^{2} \varphi_{0}\left(1+y_{1}^{\prime}\right) \\
= & \sin \theta \sin \varphi_{0} \cos \varphi_{0}\left(1+y_{1}^{\prime}\right)+\cos \theta-\cos \theta \cos ^{2} \varphi_{0}\left(1+y_{1}^{\prime}\right) \\
& \quad-\left[1-\cos ^{2} \varphi_{0}\left(1+y_{1}^{\prime}\right)\right] \\
= & \sin \theta \sin \varphi_{0} \cos \varphi_{0}\left(1+y_{1}^{\prime}\right)-\left[1-\cos ^{2} \varphi_{0}\left(1+y_{1}^{\prime}\right)\right](1-\cos \theta) .
\end{aligned}
$$

$\theta=0\left(\varphi=\pi-\varphi_{0}\right)$ means the intersection point e and the above $x_{1}$ becomes 0 as it should. Assume

$$
\theta \neq 0 \quad\left(-\pi-\varphi_{0}<\theta<0,0<\theta \leq \pi-\varphi_{0}\right) .
$$

Then, $x_{1}$ is positive if and only if

$$
\sin \theta \sin \varphi_{0} \cos \varphi_{0}\left(1+y_{1}^{\prime}\right)>\left[1-\cos ^{2} \varphi_{0}\left(1+y_{1}^{\prime}\right)\right](1-\cos \theta)
$$

that is,

$$
\frac{\sin \theta}{1-\cos \theta}>\frac{1-\cos ^{2} \varphi_{0}\left(1+y_{1}^{\prime}\right)}{\sin \varphi_{0} \cos \varphi_{0}\left(1+y_{1}^{\prime}\right)}
$$

(recall $y_{1}^{\prime} \neq-1$ and $0<\varphi_{0}<\pi / 2$ ). In other words, the $x_{1}$-coordinate is positive if and only if

$$
\cot \frac{\theta}{2}>\frac{1-\cos ^{2} \varphi_{0}\left(1+y_{1}^{\prime}\right)}{\sin \varphi_{0} \cos \varphi_{0}\left(1+y_{1}^{\prime}\right)} .
$$

Therefore, for $y_{1}^{\prime} \neq-1$ (i.e., except the circle $C_{1}$ ), the $x_{1}$-coordinate is positive for small enough $\theta>0$.

3. Main theorem. In the previous section, we introduced all preliminary lemmas for the main result of this paper. Now, we prove our main theorem.

THEOREM 3.1. If a one-to-one mapping $f: \mathbb{R}^{n} \rightarrow \mathbb{R}^{n}$ maps every unit circle onto a unit circle, then $f$ is a linear isometry up to translation.

Proof. We show $f$ preserves the distance 2. Suppose the distance between $a=f(\mathrm{~A})$ and $\mathrm{b}=f(\mathrm{~B})$ is less than 2, while the distance between $\mathrm{A}$ and $\mathrm{B}$ is 2-see Figure 3.1. Then, we show it leads to a contradiction.

Let the distance between $a$ and $\mathrm{b}$ be $2 c(0<c<1)$. Choose any unit circle $\mathrm{C}$ passing through $\mathrm{A}$ and $\mathrm{B}$ and let $f(\mathrm{C})=\mathrm{C}_{1}$. Choose a coordinate for $a$ and $\mathrm{b}$ as in Figure 3.1 such that $\mathrm{C}_{1}$ lies in the $x_{1}-x_{n}$ plane and

$$
a=\left(-1-\sqrt{1-c^{2}}, 0, \ldots, 0, c\right), \quad \mathrm{b}=\left(-1-\sqrt{1-c^{2}}, 0, \ldots, 0,-c\right) .
$$



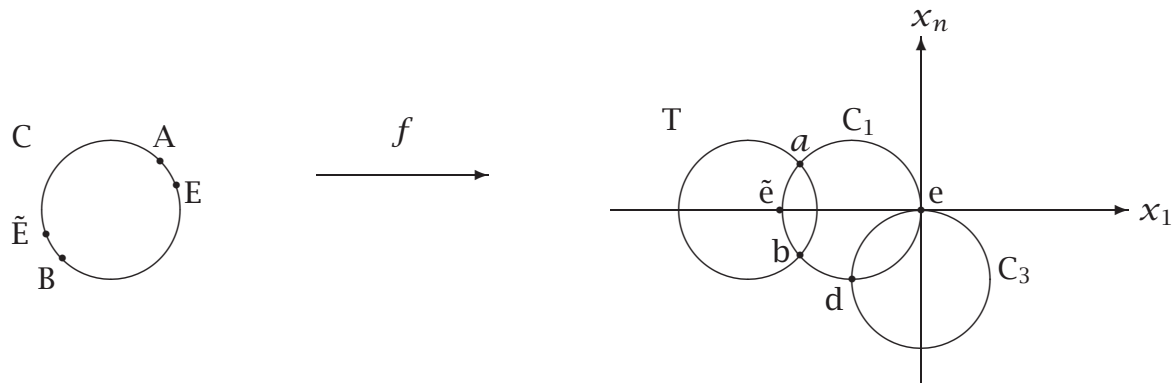

FIGURE 3.1

Let

$$
\mathrm{e}=(0, \ldots, 0), \quad \tilde{\mathrm{e}}=(-2,0, \ldots, 0) .
$$

Let $f(\mathrm{E})=$ e and $\tilde{\mathrm{E}}$ the antipodal point (in C) of E and let $f(\tilde{\mathrm{E}})=\mathrm{d}$. Let the union of all the unit circles passing through $a$ and $\mathrm{b}$ be $\mathrm{T}$ and the $(n-1)$-dimensional unit sphere passing through A and B be $S$ and the $(n-1)$-dimensional unit sphere passing through e and ẽ be $S_{1}$.

Then, it is clear that any point $\mathrm{P}$ on $\mathrm{S}(\mathrm{P} \notin\{\mathrm{A}, \mathrm{B}\})$ lies in some unit circle determined by the three points $\mathrm{A}, \mathrm{B}$, and $\mathrm{P}$. To see this, if we call $\mathrm{O}$ the common center of $\mathrm{C}$ and $\mathrm{S}$, and let

$$
\langle\overrightarrow{\mathrm{OP}}, \overrightarrow{\mathrm{OA}}\rangle=\sin \varphi_{0} \quad\left(-\frac{\pi}{2}<\varphi_{0}<\frac{\pi}{2}\right)
$$

then the unit circle determined by these three points is parameterized as

$$
\overrightarrow{\mathrm{OV}}(\varphi)=\cos \varphi\left(\frac{\overrightarrow{\mathrm{OP}}-\sin \varphi_{0} \overrightarrow{\mathrm{OA}}}{\cos \varphi_{0}}\right)+\sin \varphi \overrightarrow{\mathrm{OA}} \quad(-\pi<\varphi \leq \pi)
$$

Note that

$$
\left\{\left(\frac{\overrightarrow{\mathrm{OP}}-\sin \varphi_{0} \overrightarrow{\mathrm{OA}}}{\cos \varphi_{0}}\right), \overrightarrow{\mathrm{OA}}\right\}
$$

are orthonormal to each other and

$$
\begin{gathered}
\overrightarrow{\mathrm{OV}}\left(\varphi_{0}\right)=\overrightarrow{\mathrm{OP}}, \quad \overrightarrow{\mathrm{OV}}\left(\frac{\pi}{2}\right)=\overrightarrow{\mathrm{OA}}, \\
\overrightarrow{\mathrm{OV}}\left(-\frac{\pi}{2}\right)=-\overrightarrow{\mathrm{OA}}=\overrightarrow{\mathrm{OB}} .
\end{gathered}
$$

Since the image of this unit circle lies in T, it follows that the image of the whole $\mathrm{S}$ under $f$ lies in T. 
It is also obvious that the $x_{1}$-coordinate of any point in $\mathrm{T}$ is nonpositive. (Note that the center of any unit circle passing through $a$ and $\mathrm{b}$ has coordinate

$$
\sqrt{1-c^{2}} y-\left(1+\sqrt{1-c^{2}}, 0, \ldots, 0\right) \text { for some } y \in \mathrm{Y},
$$

(see (2.4)) and the distance between this center and any $x=\left(x_{1}, \ldots, x_{n}\right)$ is

$$
\sqrt{\left(x_{1}+1+\sqrt{1-c^{2}}\left(1-y_{1}\right)\right)^{2}+\cdots}
$$

and because

$$
\sqrt{1-c^{2}}\left(1-y_{1}\right) \geq 0
$$

positive $x_{1}$ makes the distance larger than 1 , which means that if $x_{1}>0$, we have $x \notin \mathrm{T}$.)

Now, if $\mathrm{d}=\tilde{\mathrm{e}}$, then the image of any unit circle passing through $\mathrm{E}$ and $\tilde{\mathrm{E}}$ lies in both $\mathrm{T}$ and $\mathrm{S}_{1}$. However, by Lemma 2.1, $\mathrm{T} \cap \mathrm{S}_{1}=\mathrm{C}_{1}$ and this fact contradicts the injectivity of $f$.

On the other hand, if $\mathrm{d} \neq$ ẽ, the image of any unit circle, except the circle $\mathrm{C}$, passing through $\mathrm{E}$ and $\tilde{\mathrm{E}}$ is a unit circle passing through e and $\mathrm{d}$. This unit circle is not $\mathrm{C}_{1}$ since $f$ is one-to-one, and by Lemma 2.2 it cannot stay completely in $\mathrm{T}$, a contradiction.

Consequently, $f$ preserves the distance 2. According to the well-known theorem of Beckman and Quarles, $f$ is a linear isometry up to translation.

ACKNOWLEDGMENT. This work was supported by Korea Research Foundation Grant KRF-2003-015-C00023.

\section{REFERENCES}

[1] A. D. Aleksandrov, Mappings of families of sets, Soviet Math. Dokl. 11 (1970), 116-120.

[2] F. S. Beckman and D. A. Quarles Jr., On isometries of Euclidean spaces, Proc. Amer. Math. Soc. 4 (1953), 810-815.

[3] W. Benz, Isometrien in normierten Räumen, Aequationes Math. 29 (1985), no. 2-3, 204-209 (German).

[4] _ An elementary proof of the theorem of Beckman and Quarles, Elem. Math. 42 (1987), no. 1, 4-9.

[5] W. Benz and H. Berens, A contribution to a theorem of Ulam and Mazur, Aequationes Math. 34 (1987), no. 1, 61-63.

[6] R. L. Bishop, Characterizing motions by unit distance invariance, Math. Mag. 46 (1973), 148-151.

[7] K. Ciesielski and T. M. Rassias, On some properties of isometric mappings, Facta Univ. Ser. Math. Inform. (1992), no. 7, 107-115.

[8] D. Greenwell and P. D. Johnson, Functions that preserve unit distance, Math. Mag. 49 (1976), no. 2, 74-79.

[9] S.-M. Jung, Mappings preserving some geometrical figures, Acta Math. Hungar. 100 (2003), no. 1-2, 167-175.

[10] _ Mappings preserving unit circles in $\mathbb{R}^{2}$, Octogon Math. Mag. 11 (2003), 450-453.

[11] B. Mielnik and T. M. Rassias, On the Aleksandrov problem of conservative distances, Proc. Amer. Math. Soc. 116 (1992), no. 4, 1115-1118.

[12] P. S. Modenov and A. S. Parkhomenko, Geometric Transformations. Vol. 1: Euclidean and Affine Transformations, Academic Press, New York, 1965. 
[13] T. M. Rassias, Is a distance one preserving mapping between metric spaces always an isometry? Amer. Math. Monthly 90 (1983), 200.

[14] _ Some remarks on isometric mappings, Facta Univ. Ser. Math. Inform. (1987), no. 2, 49-52.

[15] _ Mappings that preserve unit distance, Indian J. Math. 32 (1990), no. 3, 275-278.

[16] _ Properties of isometries and approximate isometries, Recent Progress in Inequalities (Niš, 1996) (G. V. Milovanovic, ed.), Math. Appl., vol. 430, Kluwer Academic Publishers, Dordrecht, 1998, pp. 341-379.

[17] T. M. Rassias and P. Šemrl, On the Mazur-Ulam theorem and the Aleksandrov problem for unit distance preserving mappings, Proc. Amer. Math. Soc. 118 (1993), no. 3, 919925.

[18] T. M. Rassias and C. S. Sharma, Properties of isometries, J. Nat. Geom. 3 (1993), no. 1, 1-38.

[19] E. M. Schröder, Eine Ergänzung zum Satz von Beckman and Quarles, Aequationes Math. 19 (1979), no. 1, 89-92 (German).

[20] C. G. Townsend, Congruence-preserving mappings, Math. Mag. 43 (1970), 37-38.

[21] A. Tyszka, A discrete form of the Beckman-Quarles theorem, Amer. Math. Monthly 104 (1997), no. 8, 757-761.

[22] _ Discrete versions of the Beckman-Quarles theorem, Aequationes Math. 59 (2000), no. 1-2, 124-133.

[23] _ A discrete form of the Beckman-Quarles theorem for rational eight-space, Aequationes Math. 62 (2001), no. 1-2, 85-93.

[24] J. Zaks, A discrete form of the Beckman-Quarles theorem for rational spaces, J. Geom. 72 (2001), no. 1-2, 199-205.

[25] _ _ The Beckman-Quarles theorem for rational spaces, Discrete Math. 265 (2003), no. 13, 311-320.

Soon-Mo Jung: Mathematics Section, College of Science and Technology, Hong-Ik University, 339-701 Chochiwon, Korea

E-mail address: smjung@wow. hongik.ac.kr

Byungbae Kim: Mathematics Section, College of Science and Technology, Hong-Ik University, 339-701 Chochiwon, Korea

E-mail address: bkim@wow.hongik.ac.kr 


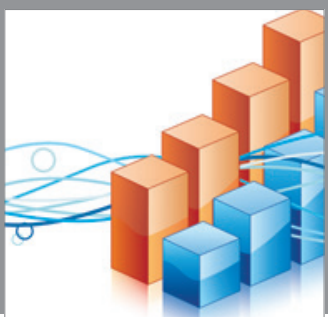

Advances in

Operations Research

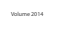

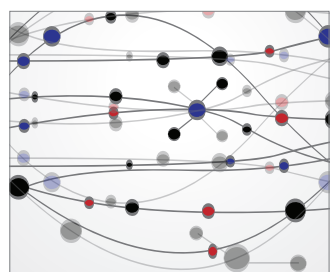

\section{The Scientific} World Journal
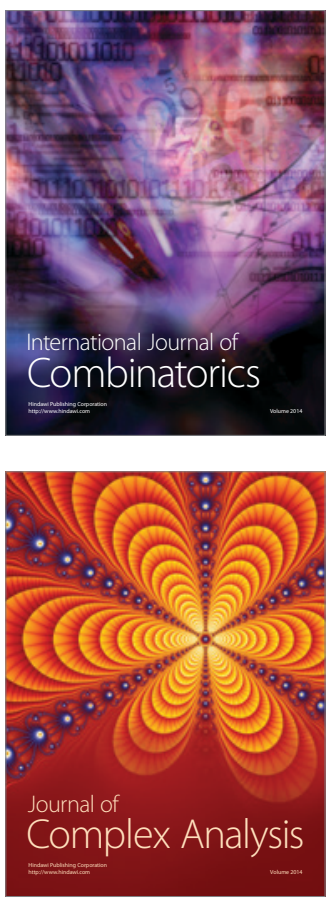

International Journal of

Mathematics and

Mathematical

Sciences
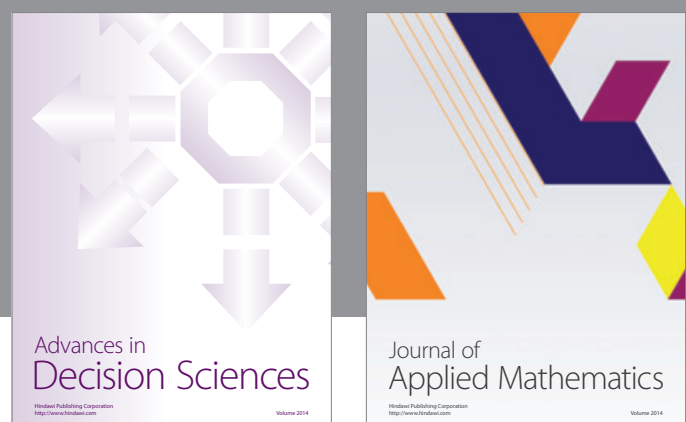

Journal of

Applied Mathematics
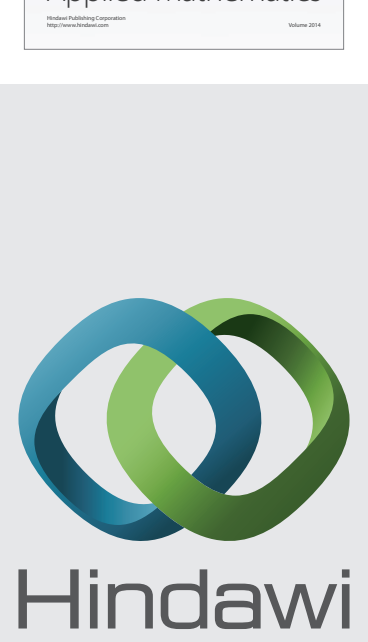

Submit your manuscripts at http://www.hindawi.com
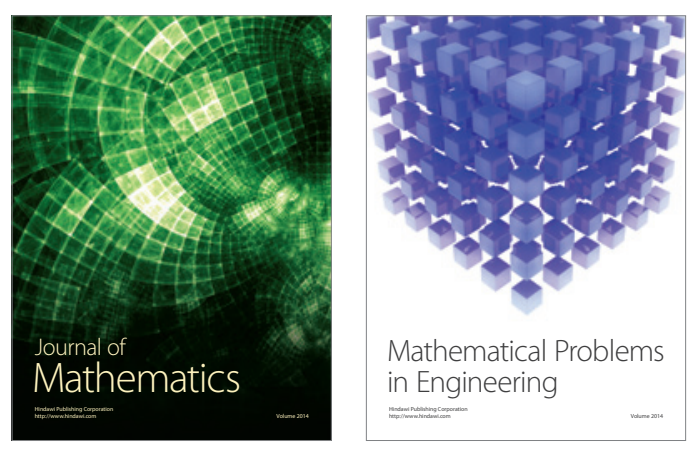

Mathematical Problems in Engineering
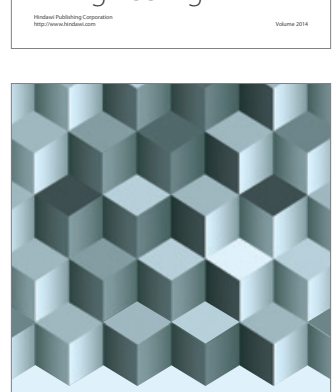

Journal of

Function Spaces
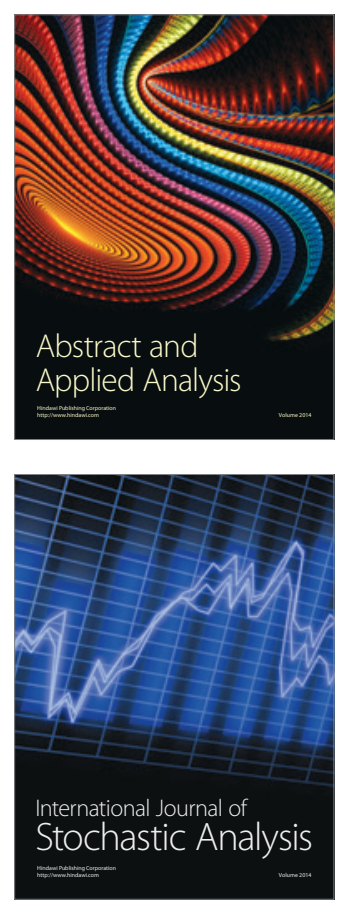

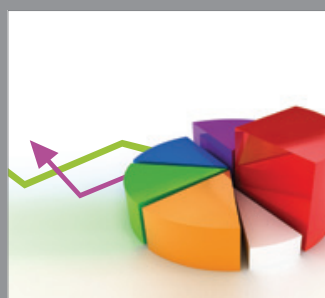

ournal of

Probability and Statistics

Promensencen
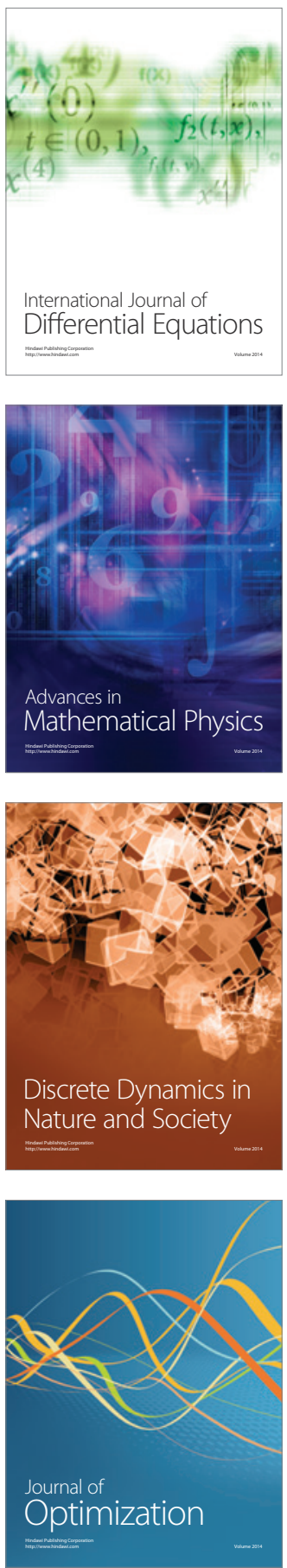